\title{
SPASTIC PARAPLEGIA IN MIDDLE AGE
}

\author{
By Colin Edwards, M.R.C.P. \\ West End Hospital for Nervous Diseases
}

Before launching into this discussion one must pause to define middle age. Obviously it is not the age period which would have been indicated $5^{\circ}$ years ago, nor is it the actual middle of the average span of life. Its limits are set by convention and they are not clear-cut, but it does not begin before 40 years of age and nowadays one can hardly say that it ends before 65 .

Paraplegia, with its serious restriction on mobility, is more dramatic and striking than paralysis of the arms, and is apt to give its name to diseases which are more than paraplegic in their effects. Hence a number of the conditions mentioned will be essentially quadriplegic and more.

Spasticity, too, although a term which connotes essentially pyramidal involvement, with its claspknife type of muscular hypertonia, can occasionally bear superficial resemblances to extra pyramidal rigidity and so some extra pyramidal diseases will have to be noted.

So much for the terms of reference.

Consideration of a clinico-pathological state can be approached with one's own idea of disease classification as a background pattern. This will ensure that one is comprehensive and is the safest method to follow if under pressure for time in an examination. Viewing one's middle-aged spastic patient as a problem in diagnosis, however, one would inevitably tend to consider the diagnosis to some extent in terms of probability, and the commoner causes would come first to mind. That is how I propose to discuss paraplegia now.

As a final prefatory remark one must stress the primary importance in neurology of history taking. The clinical signs of neurological disease are so spectacular that one can understand why students are unduly impressed by them-but in no branch of medicine is history taking more important, and it is certainly the major part of the task of diagnosis in our middle-aged paraplegic.

Disseminated sclerosis must rank as one of the commonest causes of spastic paraplegia and although four-fifths of the cases begin before middle age, it is some of these four-fifths, as well as the cases beginning in the fifth and sixth decades, that make up many of the ranks of the middle-aged spastics. The history will usually point fairly definitely to the diagnosis. Any case of early onset surviving into middle age will certainly have had a remission and there may well $\vec{\circ}$ have been subsequent exacerbations and re- $\overrightarrow{-}$ missions. Transient visual disturbances-dim- $\omega$ ness due to a retrobulbar neuritis or diplopia due $\overline{0}$ to external ocular palsies-are common in the 3 . initial attack and less so in exacerbations. And i some patients are aware of their nystagmus which ${ }_{i}$ may often be transient also. Bladder disturbances $\stackrel{\oplus}{\omega}$ - precipitancy or frequency-are also common $\overrightarrow{-}$ early symptoms. Later comes retention and $N$ usually, last of all, incontinence.

The motor symptoms of disseminated sclerosis, however, are the commonest, and particularly those involving the lower limbs. Heaviness or $\stackrel{0}{\circ}$ weakness of the legs after exertion is the most usual and it is nearly always more marked on ore $\overrightarrow{0}$ side. Where there is associated cerebellar dio turbance and the disease has not begun until the fifth, sixth or early part of the seventh decade, one has to consider the possibility of one of the cerebellar degenerations of middle life-notably delayed cortical cerebellar atrophy, and olivo-pontocerebellar atrophy, both of which show bilateral pyramidal involvement. In them, however, the curve of development of the disease is continuous and progressive, nor are ocular manifestations common-except nystagmus.

Rarely Friedrich's ataxia may show a predominance of lateral column degeneration over the posterior, and the legs may be spastic despite absence of tendon reflexes. The familial incidence, $\delta$ the foot and spinal deformities, and the lengthy history without remissions in spite of a varying $ᄋ$ rate of deterioration should differentiate these $D$ occasional cases from disseminated sclerosis. Their early onset will serve to distinguish them from the cerebellar atrophies mentioned above.

No laboratory confirmation of the diagnosis of $N$ disseminated sclerosis is possible, but about half $\underset{\omega}{N}$ of the cases on whose cerebrospinal fluid a sensitive Lange test is done show a curve of " paretic ' 0 shape or tending towards it. This is combined in about one-quarter to one-third of all cases with a $\stackrel{\oplus}{+}$ slight increase in globulin in the fluid and a raised cell count. This combination goes a long $\stackrel{\vec{P}}{\overrightarrow{0}}$ way towards establishing the diagnosis.

Of other paraplegias of inflammatory origin at $\stackrel{\overrightarrow{\mathbb{Q}}}{\stackrel{Q}{2}}$ 
this time of life those due to syphilis have perhaps been the commonest, although they are already ceasing to be so. They most commonly make their appearance late in the first half of life, and if they do appear for the first time in middle life they will be seen in its early part and not towards its end. Syphilis causes paraplegia most commonly by spinal meningo-vascular inflammation, but also occasionally by setting up a parenchymatous degeneration. The lesion in meningo-vascular cases is usually a meningomyelitis. The meningitis gives rise to pain of root origin. It occurs most commonly in the dorsal region and it may occasionally irritate the anterior roots too, causing muscular cramps or spasm. The meningitis spreads along the pial septa into the cord and its progress and resolution produce tract involvement. This process may take from a few days up to many months. Bladder disturbance is frequent and early. Spastic paralysis is often the result but quite frequently is not markedly bilateral. A bedside indication of the syphilitic nature of the spinal lesion is the tendency to patchy, cutaneous, sensory impairment, particularly of pain sensation as shown by pin prick. So it is that the victim of syphilitic meningomyelitis will sometimes show bedsores of surprising size in an area where tactile sensation is almost normal. Where a severe transverse lesion of the cord supervenes suddenly in a case with other neurosyphilitic features (e.g., root pains, impaired deep and pain sensibility and possibly pupillary changes and diminished tendon reflexes) the cause is usually the thrombosing of a spinal artery due to endarteritis. More rare types of syphilitic spastic paraplegia are those resulting from hypertrophic pachymeningitis, Erb's syphilitic spinal paralysis, gumma of the cord and syphilitic lateral and combined sclerosis.

Syphilitic hypertrophic pachymeningitis is commonest in the lower cervical region. The great dural thickening causes initially signs and symptoms due to root compression-namely pain, paraesthesiae, muscular atrophy and impaired cutaneous sensation of nerve root origin. As hypertrophy goes on the cord becomes compressed with the production first of signs of pyramidal compression (extensor plantar response and increased tendon reflexes) and later of actual slowly increasing spastic paraplegia and increasing sensory loss up to the level of the lesion.

Erb's syphilitic spinal paralysis has never received widespread acceptance, at least as a pathological entity. Clinically it shows a very slowly increasing spastic type of paraplegia with an onset ten to twelve years after the initial infection (i.e., the period of parenchymatous degenerative neurosyphilis). It may show remissions or even arrest.
It produces upgoing toes and increased tendon reflexes but spasticity and paralysis do not become marked. Sensory disturbance is slight but the bladder may sometimes be involved, and there are occasionally lightning pains. Argyll Robertson pupils are occasionally found.

It is difficult clinically to separate Erb's syphilitic spinal paralysis from syphilitic lateral sclerosis, and indeed they may well be the same condition, but at times signs of lateral sclerosis with its spastic paresis are associated with those of posterior column involvement (loss of some, at least, of the tendon reflexes, ataxia, and loss of deep sensation). The condition is then named syphilitic combined or postero-lateral sclerosis and is essentially a parenchymatous degeneration. With it may sometimes be associated muscular atrophy due to anterior horn cell degeneration.

Gumma of the cord is not common. It belongs to the meningo-vascular group of spinal syphilitic disorders and is often associated with some actual meningitis (both lepto and pachymeningitis), and with arteritis. It produces signs and symptoms of an intramedullary tumour but the spasticity reported has been most usually unilateral with contralateral sensory disturbance-the BrownSequard syndrome.

Finally, and rarely, the lower limbs may share in the general rigidity of a Parkinsonian picture of syphilitic origin, due to a parenchymatous degeneration in the striatal area, the subthalamic and tegmental mesencephalic region. These rare cases usually show associated tabes dorsalis, general paresis, or tabo-paresis.

Whenever neurosyphilis is suspected as a cause of spastic paraplegia cerebrospinal fluid analysis with Wassermann tests, and also a blood Wassermann reaction, must always be done.

In meningo-vascular cases the fluid most often shows a high cell count, usually of 30 to 200 , principally lymphocytic, although in gumma it may be low, and in pachymeningitis it may be almost normal.

The cell count in spinal parenchymatous neurosyphilis is usually only slightly raised. The Wassermann reaction may be negative in the fluid in meningo-vascular cases but is positive in the majority. It is more frequently positive still in parenchymatous cases. It should be remembered that a negative blood Wassermann reaction in association with a positive Wassermann Reaction in the cerebrospinal fluid has been estimated to occur in as many as 30 per cent. of cases of neurosyphilis, and the test may be negative in both blood and fluid in a small number (about ro per cent.) of undoubted cases. The protein is usually slightly increased in parenchymatous cases, more so in meningovascular cases, whilst hypertrophic 
pachymeningitis gives rise to a steadily rising protein culminating in a Froin's syndrome when spinal block is established. A relative excess of globulin is common. In the occasional case of rapid transverse myelomalacia due to spinal artery thrombosis, the cerebrospinal fluid may be entirely normal.

Subacute combined degeneration is a condition which is still overlooked with surprising frequency in its early stages. Its maximum incidence is round about the age of 50 , but it may begin even as early as the late twenties or as late as 70 . The principal early symptom is paraesthesiae in the feet and legs and, to a lesser extent, in the hands. Sensations as of constricting bands round the knees or of girdles round the body are also common. Weakness of the legs and general fatiguability appear next and there may be ataxia at an early stage. The signs and symptoms are symmetrical and the onset insidious. Unlike disseminated sclerosis ocular and bladder symptoms are usually absent and it begins much later as a rule than that disease. Early signs are limited to slight peripheral blunting of sensation which spreads slowly up the limbs. There may be slight ataxia and intention tremor, and there may be signs of pyramidal involvement. The blood count is seldom entirely normal, but very often it does not show a typical pernicious anaemia picture. Smoothness of the tongue is a common sign but is often so slight as to be of less diagnostic value than is commonly alleged. Achlorhydria is very nearly constant although some undoubted cases do show a slight response to histamine. Spastic paralysis is a late development in a majority of the cases, and nowadays there can be no excuse for its development. In the remainder, peripheral nerve and posterior column degeneration predominate over that in the lateral columns and a flaccid paralysis is the result. The plantar reflexes, however, are extensor. Tabes may be simulated in this second type, but serological tests and cerebrospinal fluid analysis can exclude it.

A subacute combined degeneration producing spastic paraplegia occurs in pellagra, and usually in the advanced stages, although the neurological picture is more commonly one of hypotonia and ataxia. The typical skin lesions on exposed areas and the prominence of the accompanying mental symptoms and stomatitis should suffice to settle the diagnosis. It is most likely to be missed in cases of fellagra sine pellagra and in countries where the disease is not prevalent.

Tumours of the spinal cord and canal are all characterized by a steadily progressive history and, with the occasional exception of a meningioma, they are accompanied by a rise in the cerebrospinal fluid protein. Indeed a marked rise in protein in an otherwise normal fluid is virtually pathogno-气 monic of a space-filling spinal lesion. Usual but $\frac{\pi}{\Phi}$ less constant features are motor weakness in the $\stackrel{\varrho}{c}$

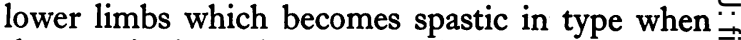
the mass is above the conus medullaris, and sensory impairment which ends abruptly at a line trans- 0 verse to the body axis. This sensory impairment 듬 is frequently dissociated in type in intramedullary $\frac{\bar{D}}{\frac{\bar{b}}{}}$ growths. Root pains at the level of the lesion are $\mathbb{Q}$ frequent in extramedullary tumours, particularly if they appear posterior to the cord, and bladder dis- $\infty$ turbance comes relatively early in many intra-. medullary growths but late in the extramedullary $\overrightarrow{\vec{\omega}}$ ones. Muscle atrophy at the segmental level of an $\stackrel{\circ}{\circ}$ intramedullary growth is not uncommon.

These are some of the salient points to be re- 3 . membered when one considers spinal cord com- N pression, but it must be emphasized that there are i very many non-neoplastic causes of this. The role of trauma is not likely to be overlooked where $\vec{v}$ spinal fracture or dislocation has occurred. Dis- N location is sometimes only momentary and spon- 음 taneously rectifies itself. This occurs principally $\vec{z}$ in the cervical region, and X-rays in such cases may show no abnormality unless the spine be $\stackrel{\widehat{\widehat{C}}}{\bar{C}}$ acutely flexed or extended for the purpose of the film-a procedure not without danger and $\$$ hardly justifiable. The cord compression in traus. matic cases is usually a momentary affair and rêf sults in laceration which shows no recovery. Laminectomy is useless in such cases. There are at least two types of acute traumatic compressions which do call for surgery, however. The first is $\propto$ where only laminae are broken and the second $\overrightarrow{\overrightarrow{0}}$ where evidence of a severe cord lesion (extensive in the transverse sense) arises soon (i.e. within a few days), but not immediately, after a spinal injury. The reasons for removing fragmented laminae are $\overline{0}$ obvious. The second group of cases includes a number in which an intramedullary haemorrhage $\frac{\dot{5}}{3}$ occurs (haematomyelia) and some in which cord compression is due to extradural haemorrhage $ᄋ$ (haematorrhachis). The cerebrospinal fluid may

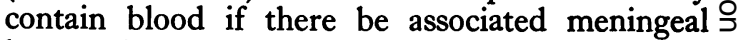
haemorrhage, but there is often a completely $\frac{D}{O}$ normal fluid. It is worth remembering, too, that there may be no hint of a subarachnoid block even $N$ though a fairly extensive haemorrhage is present. Haemorrhage into the cord can occur following $N$ very slight trauma indeed such as sudden, rapid $\mathrm{\omega}$ neck flexion, or it may be apparently spontaneous. It is more common before middle life, however. It occurs principally in males and usually where spinal vascular abnormality is already present. In ? such cases of cord compression laminectomy should be done. i he results of clot removal are sometimes most gratifying.

Late effects of trauma which may produce cord 
compression are thecal scarring, herniation of an intervertebral disc and arachnoiditis with cyst formation (meningitis serosa circumscripta). All three conditions, if diagnosed early enough, can be helped considerably by surgery. The traumatic history should put one on the alert although the remainder of the story is not usually distinguishable from that of any slowly increasing spaceoccupying lesion. Thecal scarring virtually never occurs in the absence of easily recognizable preceding bony injury. Because of their position near the intervertebral foramina, where they impinge on nerve roots, disc herniations are seldom painless, but when they cause cord lesions this is due principally to interference with the cord blood supply, sometimes associated with arachnoiditis, and not to direct structural deformation of the cord itself by the hernia. Cyst-forming arachnoiditis is seldom painful. lt may be very slow in development and spastic spinal symptoms are more prominent than the sensory ones. It is a condition, of course, which may arise from many causes other than trauma-notably local spinal infections, general infections such as the specific fevers, syphilis, any acute meningitis, and secondary to spinal cord disease such as syringomyelia or disseminated sclerosis. The bulk of cases of spinal arachnoiditis, of course, are not characterized by cord compression, and many of the post-meningitic spastic states are due to a meningo-myelitis rather than to cord compression. In syphilis, hypertrophic pachymeningitis, as already mentioned, may give rise to cord compression.

Other inflammatory causes of cord compression with spastic paraplegia are spinal caries and extradural abscess. This is most commonly tuberculous, but it may be due to one of the common pyogenic organisms. In the latter group the patient is usually acutely ill with septicaemia and the diagnosis will probably prove obvious, but in the tuberculous cases bony disease may not be apparent at a stage when cord symptoms are quite definite. The area of the spine near the cord lesion must be watched assiduously for signs of the inflammation which the patient's temperature, erythrocyte sedimentation rate and general condition will probably suggest. Tenderness of the spine is likely to be the first sign.

Frank Pott's disease of the spine need give rise to no difficulty, and although it is commonest in the young it is also found in middle age and even late in life.

Rarely a hydatid cyst may form within the spinal canal and compress the cord, but this is almost a curiosity even in countries where the disease is oummon.

Cord compression by acute meningeal extensions of Hodgkin's disease or acute leukaemic tumours need offer no difficulty in diagnosis because of signs of these diseases elsewhere. Both conditions are much commoner before middle age and they will be very rare causes of spastic paraplegia in the middle aged.

However it is neoplastic disease proper which is by far the commonest cause of extradural cord compression, and this is usually secondary carcinoma invading bone. Primary growths are usually sarcomata.

In every case of spinal cord disease where a diagnostic lumbar puncture is done a manometric test for the freedom of subarachnoid fluid flow must be made, using jugular vein compression to raise the fluid pressure in the head. The absence of a block does not exclude a space-filling lesion, but if present it goes a long way towards establishing such a diagnosis. Bedside examination alone is often insufficient to localize the lesion within limits sufficiently narrow for a surgical approach. Myelography must then be used and a tilting table with facilities for screening the recumbent patient are desirable. It is only by screening that many partial blocks can be detected. The radio-opaque medium used can be introduced by the lumbar route and allowed to run upwards or by the cisternal route and allowed to gravitate downwards. If the lumbar route is used care must be taken that substances such as lipiodol do not get up into the cranial cavity as the iodine may separate out and set up arachnoiditis by irritation. No more than 2 cc. of lipiodol is necessary, and the stability of the preparation is very important. Indeed it is desirable, as a rule, to try to avoid putting lipiodol into the theca unless there is a reasonable prospect of its subsequent removal at operation.

Another group of spastic paraplegics is that due to acute myelitis other than syphilitic. These are almost all sudden in onset. Those which are due to direct extension from a vertebral abscess present little difficulty in diagnosis. Many cases of acute transverse myelitis, however, show no obvious cause. Their onset is rapid, even sometimes with the speed of a stroke, and although they may complicate infectious illness such as smallpox or measles, and although some follow hard upon the giving of a spinal anaesthetic, the aetiology of many is remarkably obscure. The cell count in the fluid tends to be abnormally high and lymphocytic in type, and although virus infection has been blamed for many of these cases this is an unproven suspicion as yet.

Most of these cases of acute myelitis occur early in adult life but they are not rare in the middle aged.

Sometimes the acute cord lesion is part of a disseminated encephalomyelitis which may com- 
plicate one of the exanthemata, may follow vaccination, or may arise quite independently. Spastic paralysis is rare from such a cause, however, most patients showing flaccidity during the acute state and, if they survive, recovery tends to be well nigh complete.

These acute myelitides may be simulated by sudden cord compression due to vascular mishaps such as haemorrhage into a vascular tumour, or a syringomyelic cavity, or by spontaneous haematomyelia. Cerebrospinal fluid analysis will usually serve to distinguish these from the inflammatory cases where the cell count is almost always raised and where subarachnoid block is uncommon. The preceding history, of course, often supplies the answer.

Syringomyelia perhaps merits a little special consideration. The disease will almost always have declared itself, although sometimes very unobtrusively, before middle age, usually first in the sensory field with dissociated loss over a hand or arm and extending on to the neck and thorax. Although most cases show signs of pyramidal tract involvement on one or both sides, only a few develop spastic paraplegia, but this development has occurred after the age of 40 , either due to the steady enlargement of the cavity in the cord or to its very occasional rapid distension by haemorrhage. A spinal block may be set up and even a transverse cord softening. Syringomyelic cases showing extensive cord tract involvement with a complete or material degree of spinal block merit a laminectomy. Incision of the cavity wall in the mid-posterior line with consequent relief of pressure may lessen the upper motor neurone paralysis considerably.

Perhaps next one should remember those degenerative cord diseases of quite unknown originthe abiotrophies-which not infrequently cause spastic paraplegia in the middle aged. The most sinister is amyotrophic lateral sclerosis, painless, insidious and lethal. It is essentially a disease of middle life. Muscular atrophy commonly beginning in the hand intrinsics is accompanied by muscular fasciculation that is frequently widespread, with increased tendon reflexes (in contrast with the syphilitic kind) and pyramidal involvement that sometimes produces very spastic legs. As a general rule the more spastic the case the less is the atrophy and the slower the disease. Objective sensory changes are absent except for occasional blunting of cutaneous sensibility round the periphery of limbs, due possibly to impoverished circulation there. As in all the abiotrophies, cerebrospinal fluid analysis is normal. It is worth emphasizing, whilst talking of amyotrophic lateral sclerosis, that muscular twitchings sometimes of a fibrillary or fascicular kind, often occur in young, middle aged and elderly subjects气 in the absence of any hint of muscular atrophy or of lateral sclerosis. They are provoked in some by fatigue and in others by smoking, but in many subject no cause is apparent. The label 'myokymia ' has been given to certain varieties of these.0 One should never rush into a diagnosis of amyo-흠 trophic lateral sclerosis merely because of the presence of such twitchings. In doubtful cases $\mathbb{\Phi}$ the electromyogram can give useful help in theo differential diagnosis. Pure primary lateral sclerosis? without atrophy of muscles (except ultimately. from disuse) is an entity whose separate existence $\vec{\omega}$ is denied by many neurologists. It is contendedo that these are cases of amyotrophic lateral sclerosiso beginning in the pyramidal sphere and that if 3 . watched long enough amyotrophy will usually N appear, or, if examined with sufficient care post-î mortem, typical anterior horn cell changes will bee revealed. The surprising frequency, however, $\overrightarrow{-}$ with which one sees such cases in the absence of any vascular or syphilitic basis has raised my own? suspicion that there does exist a primary abio- $-\vec{r}$ trophic lateral sclerosis, and one recent personalo case in a man of 60 with spastic paraplegia, no $\frac{\bar{S}}{3}$ accompaniments and a normal fluid, blood, gastric $\overrightarrow{.}$ secretion and vascular system, appears to hagec become statir after nearly three years.

There is, of course, a progressive bilate pyramidal involvement without muscular atrophy, which is due largely to cerebral atheroma. Theo degree of paraplegia in these cases is usually mildœ and a pseudo-bulbar type of spastic dysarthria $\mathbb{D}$ commonly accompanies it. This speech impair- $\overrightarrow{\overrightarrow{0}}$ ment is due to bilateral pyramidal tract impairment 3 above the level of the speech nerve nuclei. Cerebrospinal fluid protein in such vascular cases is often? slightly increased (e.g. to 60 to $80 \mathrm{mgm}$./ $100 \mathrm{cc}$.) in contradistinction to the two preceding types.

Another abiotrophic type is heredo-familialo spastic paralysis which begins in the first half of life (although often not until the third or even fourth decade), but whose progress is usually remarkably slow, many of the victims living on to을 old age. It commences in the legs and it may be $\rightarrow$ as much as 20 years before the arms are involved. There is no muscular atrophy and no sensory N change but the degree of spasticity is often very severe.

Another ' pure' spastic paraplegia often persisting into middle and old age from infancy is that of cerebral diplegia (which includes Little's disease). Involvement of the upper limbs also occurs and some of the cases are associated with ${ }^{+}$ athetosis due to striatal damage. These cases show $\frac{7}{0}$ no deteriroration granted normal care, and con- $\frac{\vec{O}}{\mathbb{D}}$ siderable benefit from patient education is possible. $\frac{\stackrel{\rho}{\mathbb{Q}}}{\mathbb{Q}}$

The rigidity of striatal disease, whether of $\stackrel{\mathbb{Q}}{\sigma}$ 
Parkinsonian degeneration or encephalitis, can hardly be said to cause paraplegia, and never true spasticity.

Final consideration must be given to hysteria. Hysteria does not make its initial appearance in middle-aged subjects with a hitherto stable psychosomatic history, and only the rash will diagnose it at this time of life if there is no trace of a previous neurotic diathesis. It is not uncommon, however, to see hysterical paraplegia in middle life. The diagnosis is only difficult at the bedside in very occasional cases where plantar reflexes cannot be elicited and the characteristic non-anatomical sensory loss of hysteria is absent. These are few, however, and the absence of an extensor plantar response in a severe spastic paralysis nearly always clinches the diagnosis. On the sensory side it is always well to remember that the buttock and perianal skin areas are supplied through the lower sacral nerves from a cord level that is much lower than that receiving sensation from the foot and leg. Yet quite commonly the hysterical paraplegia which mimics a severe cord lesion will show profound loss of pin-prick sensation in the legs with normal buttock sensation. A carefully taken history alone, however, usually takes one nearly all the way to the diagnosis in hysterical paraplegics.

It has not been possible in this article to do more than outline a few of the salient diagnostic points in spastic paraplegia in middle life. To discuss treatment has been impossible. I would like to close, however, with an important therapeutic generalization. Acute inflammation, haemorrhage or shock in the central nervous system as elsewhere in the body requires rest as the cornerstone of treatment. Where C.N.S. function is impaired from any other cause, however, rest, except for the purpose of recuperation from fatigue, is contraindicated. This point is very important in paralysis in the young, but in middle life and after it is vital.

\section{BOOK REVIEWS}

\section{TUMOURS OF BONE}

By Charles F. Geschickter, M.D., and Murray

M. Copeland, M.D. 3rd Edition. Pp. xviii + 810, with 642 illustrations. London: J. B. Lippincott. I 949 . 5 guineas.

This book is a unique work for reference in so far as it tabulates the clinical, radiological, pathological and prognostic findings in a truly enormous number of cases of bone tumours. It is beautifully produced and almost over lavishly illustrated. As in the previous editions each type of tumour is dealt with in all its aspects, illustrated by a summary of case records, many hundreds in number, a chart of age incidence, a skeletal diagram of site incidence and numerous photographs of X-rays, macroscopic and microscopic specimens. In addition, the authors make a detailed and persistent effort to correlate the tumour picture with disorder of varying phases of bone development; at times carried to illogical extremes, of which the following statement (p. I I I) is an example:- ' The clinical features of primary chondrosarcoma reflect in part the primitive histogenesis of this neoplasm. The frequency with which Negroes are affected (approximately I 5 per cent.) is unusual among the osteogenic sarcomas as a group, and suggests a lower evolutionary form of osteogenesis for this tumour.'
The style is heavy and at times not lucid; repetitions and summaries, however, do in most instances clarify the authors' ideas. Certain sections should have been omitted from this edition, in particular the two introductory chapters by Dean Lewis and Bloodgood. Their unassailable dicta that physical examination, roentgenographs, biopsies and blood Wassermann examinations are diagnostically helpful are amply brought out in the later text and in any case have long been universally appreciated. The chapter on endocrinopathies is too short to serve any useful purpose. Table 76, listing the sites of primary carcinoma which have given rise to osseous metastases, has not been altered since the first edition in I93I and in our view underestimates the role of bronchial carcinoma. The table cites a total of 334 cases of secondary bone carcinoma of which only 4 came from a primary in the bronchus in contrast to 134 from prostate, 100 from breast and 22 from kidney. However, table 72 details the sites of metastases from bronchial carcinoma in 3,212 autopsied cases. The numerous misprints, contradictions and repetitions lay this book open to easy criticism, but they do not detract from the fact that it summarizes the findings in an impressive collection of cases and in contrast to many textbooks proves really helpful to the reader searching a factual answer to a concrete problem.

I.D. 